\title{
Variability of Normal Pressure Hydrocephalus Imaging Biomarkers with Respect to Section Plane Angulation: How Wrong a Radiologist Can Be?
}

\author{
(D) P. Ryska, (D) O. Slezak, (D) A. Eklund, (D). Salzer, (D). Malm, and (D). Zizka
}

\section{ABSTRACT}

BACKGROUND AND PURPOSE: Systematic analysis of angulation-related variability of idiopathic normal pressure hydrocephalus imaging biomarkers has not been published yet. Our aim was to evaluate the variability of these radiologic biomarkers with respect to imaging plane angulation.

MATERIALS AND METHODS: Eighty subjects (35 with clinically confirmed idiopathic normal pressure hydrocephalus and 45 age- and sex-matched healthy controls) were prospectively enrolled in a 3T brain MR imaging study. Two independent readers assessed 12 radiologic idiopathic normal pressure hydrocephalus biomarkers on sections aligned parallel or perpendicular to the bicallosal, bicommissural, hypophysis-fastigium, and brain stem vertical lines, respectively.

RESULTS: Disproportionately enlarged subarachnoid space hydrocephalus, simplified callosal angle, frontal horn diameter, z-Evans Index, and cella media vertical width did not show significant systematic differences in any of 6 section plane combinations studied. The remaining 7 biomarkers (including the Evans Index and callosal angle) showed significant differences in up to 4 of 6 mutually compared section plane combinations. The values obtained from sections aligned with the brain stem vertical line (parallel to the posterior brain stem margin) showed the most deviating results from other section angulations.

CONCLUSIONS: Seven of 12 idiopathic normal pressure hydrocephalus biomarkers including the frequently used Evans Index and callosal angle showed statistically significant deviations when measured on sections whose angulations differed or did not comply with the proper section definition published in the original literature. Strict adherence to the methodology of idiopathic normal pressure hydrocephalus biomarker assessment is, therefore, essential to avoid an incorrect diagnosis. Increased radiologic and clinical attention should be paid to the biomarkers showing low angulation-related variability yet high specificity for idiopathic normal pressure hydrocephalus-related morphologic changes such as the z-Evans Index, frontal horn diameter, or disproportionately enlarged subarachnoid space hydrocephalus.

ABBREVIATIONS: $\mathrm{BVR}=$ brain-to-ventricle ratio; $\mathrm{CA}=$ callosal angle; $\mathrm{CMW}=$ cella media vertical width; $\mathrm{CTR}=$ cella media-to-temporal horn ratio $\mathrm{DESH}=$ disproportionately enlarged subarachnoid space hydrocephalus; El = Evans Index; FHD = frontal horn diameter; FHVD $=$ frontal horn vertical diameter; $\mathrm{HC}=$ healthy control; Hy-Fa = hypophysis-fastigium; iNPH = idiopathic normal pressure hydrocephalus; ISD = inner skull diameter; MSID = maximum supratentorial intracranial diameter; PBSM = posterior brain stem margin; simpCA = simplified callosal angle; SVW = supraventricular brain vertical width; THW = temporal horn vertical width; ZEI = z-Evans Index

$\mathbf{T}$ he prevalence of idiopathic normal pressure hydrocephalus (iNPH) may be as high as $0.5 \%$ in the population older than 65 years of age, and iNPH has emerged as a significant health issue for the aging population in developed countries. The

Received July 2, 2020; accepted after revision January 13, 2021.

From the Department of Diagnostic Radiology (P.R., O.S.), University Hospital Hradec Kralove, Hradec Kralove, Czech Republic; Department of Diagnostic Radiology (O.S.), Charles University, Faculty of Medicine, Hradec Kralove, Czech Republic; and Departments of Radiation Sciences (A.E.), Pharmacology and Clinical Neuroscience (J.S., J.M.), Section of Neurology, and Imaging and Functional Medicine (J.Z.), University Hospital of Umeå, Umeå, Sweden.

Please address correspondence to Jan Zizka, MD, University Hospital of Umeå, Umeå, Sweden; e-mail: jan.zizka@regionvasterbotten.se

- Indicates open access to non-subscribers at www.ajnr.org

http://dx.doi.org/10.3174/ajnr.A7095 symptoms include gait disturbance, memory impairment, and urinary incontinence. Underdiagnosed iNPH entails missed opportunities for successful ventricular shunt treatment. ${ }^{1,2}$

Numerous linear, angular, and volumetric measurements; relative indexes; CSF flow studies; as well as visual semiquantitative or qualitative parameters have been proposed as radiologic biomarkers of iNPH..$^{3-15}$ Most of the iNPH imaging biomarkers have originally been defined by their authors using arbitrary or proprietary settings in terms of defining section planes and/or measurement techniques, which, in many cases, do not comply with the routines of daily radiologic practice. This issue particularly applies to the use of the bicommissural plane, which is rarely used in routine practice because the anatomic landmarks are subtle and, therefore, difficult to apply. 


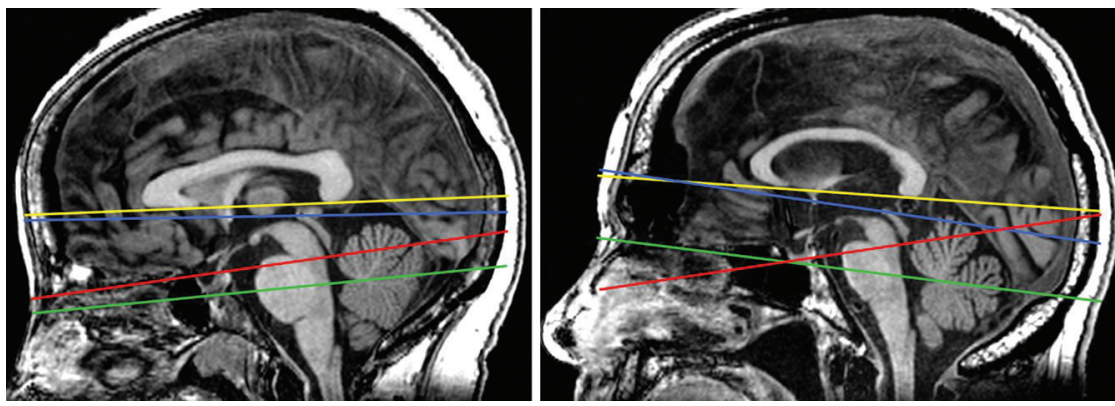

FIG 1. Definition of standard transverse imaging planes: bicallosal (yellow), bicommissural (blue), Hy-Fa (green), and perpendicular to the posterior brain stem margin (red), shown on midsagittal MR images of 2 different individuals. Note the differences in their mutual alignment between the 2 subjects.

categorized as having probable iNPH, and $11 \%(4 / 35)$, as having possible iNPH. Subjects with significant neurologic comorbidities or a known etiology of NPH (secondary NPH) were not included in the study.

The healthy control group comprised sex- and age-matched volunteers primarily recruited through an advertisement in a local newspaper who showed no signs of neurologic, psychiatric, or advanced atherosclerotic disease on a subsequent clinical examination performed by a neurologist. We applied the following exclu-

Various institutions have been using different definitions of standard imaging planes for decades. For example, a "standard" transverse CT or MR imaging plane can be angulated according to at least 5 different section definitions. Such interinstitutional differences may lead to different outcomes of iNPH imaging studies.

The frequently used Evans Index (EI, originally defined on pneumoencephalograms in 1942) was not initially intended for use with cross-sectional imaging. ${ }^{16}$ The Evans Index was secondarily adopted for transverse CT sections aligned parallel to orbitomeatal line in 1976 and subsequently transferred into MR imaging, without a particular respect for the transverse plane definition. ${ }^{17}$ Even recently published studies often do not precisely define anatomic landmarks used for angulation of transverse or coronal sections in the methodology section. Such methodologic inconsistencies might have contributed to widely differing diagnostic performances of iNPH biomarkers reported by various authors. $^{3-5,8-12}$

A systematic evaluation of the variability of iNPH biomarkers related to imaging plane definition (bicallosal, bicommissural, hypophysis-fastigium [Hy-Fa], brain stem vertical line, and so forth) has not been published so far. The aim of this study was to assess the variability of individual iNPH cross-sectional imaging biomarkers with respect to different imaging plane angulations.

\section{MATERIALS AND METHODS \\ Study Population}

Eighty subjects were prospectively enrolled in the study: 35 subjects with clinically confirmed iNPH ( 25 men and 10 women; mean age, 74.0 [SD, 7.2] years) and 45 sex- and age-matched healthy controls (HCs: 32 men and 13 women; mean age, 72.6 [SD, 5.7] years).

Patient history, neurologic objective findings, and brain $\mathrm{MR}$ imaging were used for diagnosing iNPH in accordance with the international iNPH guidelines. ${ }^{18}$ Six subjects with iNPH (17\%) showed signs of dementia (Mini-Mental State Examination scores, 20-24 points), and 15 subjects (43\%) had mild cognitive impairment (Mini-Mental State Examination scores, 24-27 points). All patients except one were able to walk $10 \mathrm{~m}$ with or without an assistive device. In addition, all patients underwent a CSF dynamic investigation (including estimation of CSF outflow resistance) and a short-term tap test, which had positive findings in 19 patients (54\%). Eighty-nine percent of patients (31/35) were sion criteria: medications influencing the central nervous system or blood coagulation; electrocardiogram evidence of arrhythmia, left ventricular hypertrophy, or myocardial infarction; a history and/or clinical or radiologic signs of neurologic disease (including ventriculomegaly, stroke, tremor, ataxia, walking/balance difficulties); significant cardiovascular disease; diabetes mellitus; $\geq 2$ ancillary vascular risk factor (smoking, hypertension, hyperlipidemia); ongoing infectious disease; known serious disease that may reduce life expectancy; and contraindications to MR imaging. Cognition, balance, and gait were tested using the Mini-Mental State Examination ( $>27$ points required) and the one-legged balancing test (30 seconds), tandem stand (10 seconds), Timed Upand-Go test, and 300-m walk test. Of 59 screened subjects, 50 passed the clinical evaluation and underwent brain MR imaging. Five subjects were subsequently excluded from participating in the study due to MR imaging findings of cortical infarctions $(n=3)$, cerebellar tonsillar herniation $(n=1)$, and subdural hygroma $(n=1)$. Thus, the HC group consisted of 45 individuals.

The study was approved by the Umeå University institutional review board. Signed informed consent was obtained from all participants of the study.

\section{MR Image Acquisition and Analysis}

All subjects were examined on a $3 \mathrm{~T}$ MR imaging system by means of a 3D T1-weighted spoiled gradient-echo imaging sequence with a voxel size of $0.49 \mathrm{~mm}^{3}$. Multiplanar reformats parallel and perpendicular to the bicallosal, bicommissural, Hy$\mathrm{Fa}$, and brain stem vertical line were obtained.

Anonymized MR imaging data were independently evaluated by 2 observers: a senior radiology resident with 5 years of radiology training and a neuroradiologist with 21 years of clinical experience. Both readers were blinded to clinical data.

\section{Definition of Anatomic Planes on Midsagittal Sections}

Inferior margins of the rostrum and splenium of the corpus callosum were used as anatomic landmarks for defining the bicallosal plane (Fig 1). The anterior and posterior commissure defined the bicommissural plane. The inferior pituitary gland margin and the apex of the fourth ventricle defined the hypophysis-fastigium (Hy-Fa) plane. The brain stem vertical line (ie, fourth ventricle floor plane) was parallel to the posterior brain stem margin (PBSM). 


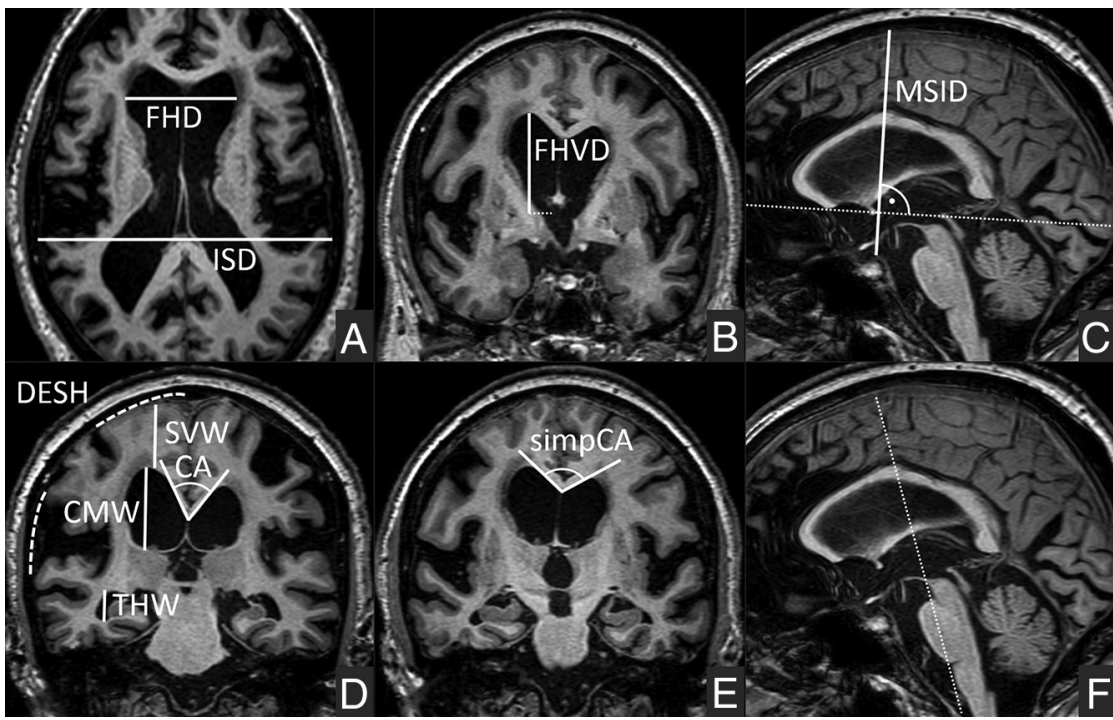

FIG 2. Graphic representation of biomarker measurements. $A$, Maximum frontal horn diameter (FHD) and inner skull diameter (ISD). B, Frontal horn vertical diameter (FHVD) measured to the midpoint of the foramen of Monro. C, Maximum supratentorial intracranial diameter (MSID) measured perpendicular to the bicommissural line (dotted line). D, Maximum vertical widths of the supraventricular brain (SVW), cella media (CMW), and temporal horn (THW), respectively; callosal angle (CA); and DESH (curved dashed lines, rated as severe, grade 2 in this case) measured on the coronal section passing through the posterior commissure. E, Simplified callosal angle (simpCA) measured at the corpus callosum midpoint on the coronal section paralleling the PBSM ( $F$, dotted line). Note that for each biomarker, 4 measurement values have been obtained from 4 different sections (as defined in Fig 1). To keep the illustration simple, we show measurements on sections aligned parallel and perpendicular to the bicommissural line, except for the simpCA ( $E$ and $F$ ). For details, see the Materials and Methods section.
Disproportionately enlarged subarachnoid space hydrocephalus (DESH) referred to visual semiquantitative assessment of the disproportion between enlarged volumes of subarachnoid spaces at the Sylvian fissure and decreased volumes at the superior parasagittal convexity on the respective coronal sections passing through the posterior commissure. ${ }^{19,20}$ DESH was rated as follows: no disproportion $=0$, mild-to-moderate disproportion $=1$, severe disproportion $=2$ (Figs 2 and 3). A single enlarged sulcus in otherwise reduced subarachnoid spaces did not alter the rating because it was known to be a relatively common feature of iNPH. ${ }^{19}$ In rare cases when the Sylvian fissure was narrower than the parasagittal convexity sulci, findings were rated as -1 or -2 , respectively.

The callosal angle (CA) was defined by 2 lines tangentially aligned with the superior-medial walls of the lateral ventricles on the coronal sections passing through the posterior commissure. ${ }^{5}$ The simplified callosal angle (simpCA) was assessed on the coronal sections passing through the corpus callosum midpoint, which was

\section{iNPH Biomarker Definitions}

Ten previously published radiologic measures as well as 4 calculated indexes ${ }^{5-7,12,16-21}$ were individually evaluated on transverse/coronal imaging sections of 4 different angulations (bicallosal, bicommissural, Hy-Fa, and PBSM) (Fig 2): The frontal horn diameter (FHD) was defined by the maximum distance between the lateral margins of the frontal ventricular horns on the respective transverse sections. ${ }^{16,17}$ The inner skull diameter (ISD) was defined by the maximum lateral-to-lateral distance between the inner laminae of the parietal bones on the same transverse sections that were used for FHD measurements. ${ }^{16,17}$ The frontal horn vertical diameter (FHVD) was defined by the maximum z-axis (vertical) diameter of the frontal horns measured at the level of the foramen of Monro, ie, the vertical distance between the ceiling of the lateral ventricle and the midpoint of the foramen of Monro on 4 coronal sections. ${ }^{7}$ The maximum supratentorial intracranial diameter (MSID) was measured bone-tobone on the midsagittal section; the measurement line was drawn

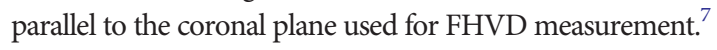

The maximum vertical width of the supraventricular brain (SVW; width of brain tissue superior to the ventricle), the cella media of the lateral ventricle (CMW), and the temporal horn (THW) were measured on 4 coronal sections passing through the posterior commissure (modified from Yamada et $\mathrm{al}^{12}$ ). Depending on the degree of ventricular dilation, the THW was measured either superior to the hippocampus only (if the hippocampus completely covered the floor of the temporal horn) or superior + lateral + inferior to the hippocampus (if the dilated temporal horn extended below the level of the hippocampus). identified on the midsagittal section. The angle was drawn with the vertex placed in the inferior point of the corpus callosum and the sides tangential to the lateral ventricles. ${ }^{6}$

In addition, 4 calculated indexes (ratios) derived from the above-mentioned linear measurements were assessed on 4 differently angulated transverse/coronal sections: The Evans Index (EI) was defined as the FHD divided by the ISD obtained from the same section. ${ }^{16,17}$ The $z$-Evans Index (ZEI) was defined as the FHVD divided by the MSID. ${ }^{7}$ The brain-to-ventricle ratio (BVR) was defined as the ratio between the SVW and the CMW. ${ }^{12}$ The cella media-to-temporal horn ratio (CTR) was defined as the ratio between the CMW and THW. In cases of ventricular asymmetry, measurements.

Note that the inner skull diameter and maximum supratentorial intracranial diameter were neither considered nor evaluated as iNPH biomarkers, yet they were prerequisites for calculating the EI or ZEI, respectively.

\section{Data Analysis}

Interrater agreement was studied by means of the intraclass correlation coefficient for a 2-way random effects model and absolute agreement. Correlation coefficient analysis of each biomarker was based on measurements from all 4 planes, giving a total of $n=320$ $(4 \times 80)$ measurements per biomarker. Results of the reliability analysis were presented as intraclass correlation coefficients with 95\% confidence intervals for 2 independent raters. the hemisphere with a larger cella media was used for all 


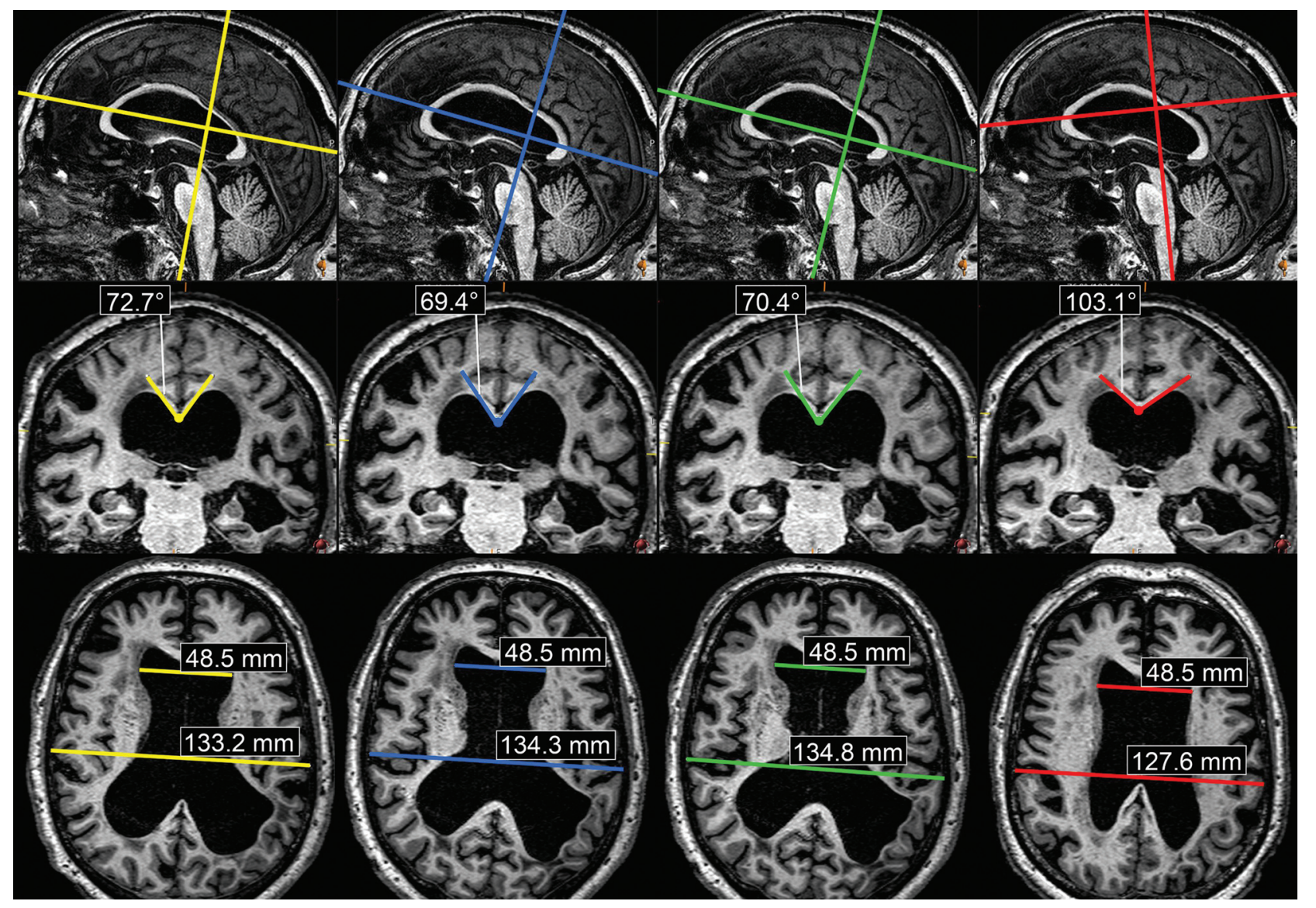

FIG 3. Variability of the CA (middle row) and El (lower row) as measured on bicallosal (yellow), bicommissural (blue), Hy-Fa (green), and PBSM (red) sections of a single subject with iNPH. The CA yields $73^{\circ}, 69^{\circ}, 70^{\circ}$, and $103^{\circ}$ in the 4 respective planes (maximum percentage difference of $39.5 \%$ ). The El shows a less variable range of values: $0.364,0.361,0.359$, and 0.380 , respectively (maximum difference of $5.7 \%$ ). Color lines on the sagittal sections (upper row) refer to the alignment of the respective coronal and transverse sections below. The disproportion between Sylvian and parasagittal convexity subarachnoid spaces (DESH) has been rated moderate in this case (grade 1).

To assess whether individual biomarkers showed systematic differences when measured on sections with different angulations, we used 2-way repeated measures ANOVA (Huynh-Feldt correction) with angulation as a within-subjects factor and disease status (iNPH/ $\mathrm{HC}$ ) as a between-subjects factor, including the angulation by disease status interaction term (angulaton iNPH/HC). For biomarkers that showed statistically significant dependence on angulation in the repeated measures ANOVA, we analyzed the pair-wise comparison among angulation planes. Means \pm standard errors of paired differences were calculated and presented $(n=80)$ for the paired tests that were significant. A Bonferroni correction was used for the pair-wise comparisons. The level of statistical significance was set to $P<.05$.

\section{RESULTS}

The interrater agreement analysis yielded reliable agreement for DESH (intraclass correlation coefficient $=0.851$; 95\% CI, $0.752-$ 0.903) and excellent agreement for the remaining 11 biomarkers (intraclass correlation coefficients ranging from 0.963 to 0.997 ; $95 \%$ CI within \pm 0.011 for all biomarkers). Because all intraclass correlation coefficients were highly significant $(P<.001)$, averaged values from both raters were used in all subsequent analyses.

When we compared the values of individual biomarkers obtained from differently angulated sections, DESH, simplified CA,
FHD, ZEI, and CMW did not show significant systematic differences in the repeated measures ANOVA (Table 1, column 5: "Angulation"). The remaining 7 biomarkers showed significant differences between up to 3 (eg, EI) or even 4 (eg, CA) mutually compared section pairs (Table 2). The biomarker values obtained from sections aligned parallel or perpendicular to the fourth ventricle floor (PBSM) showed the most deviating results from other section angulations. The lowest biomarker variability was found when comparing the results obtained from those section pairs: bicallosal and $\mathrm{Hy}-\mathrm{Fa}$ or bicommissural and Hy-Fa; these combinations of sections significantly differed in a single biomarker only (BVR or THW, respectively; see Table 2, columns 3 and 5).

The angulation by the iNPH/HC interaction term was significant for the THW and CA, ie, dependence on angulation differed between the iNPH and HC groups for the THW and $\mathrm{CA}$ only, with the effects being larger for iNPH in both cases (Table 1, column 6: "Angulation iNPH/HC"). Differences of biomarker values between the iNPH and HC groups were significant $(P<.001)$ for all 12 biomarkers evaluated (Table 1 , column 4: “iNPH/HC”).

Graphic examples of the variability of the EI and CA measured on 4 standardized imaging sections of the same subject are presented in Fig 3. 
Table 1: Variability of iNPH biomarkers with respect to imaging plane angulation ${ }^{\mathrm{a}}$

\begin{tabular}{lcccccc}
\hline & \multicolumn{2}{c}{ Mean } & & \multicolumn{3}{c}{ Repeated Measures ANOVA (P Values) } \\
\cline { 2 - 3 } \cline { 5 - 6 } Biomarker & HC & iNPH & & iNPH/HC & Angulation & .463 \\
DESH & 0.12 & 1.31 & $<.001$ & .264 & .463 \\
simpCA & 129.8 & 98.2 & $<.001$ & .151 & .950 \\
FHD & 39.1 & 51.2 & $<.001$ & .075 & .757 \\
ZEI & 0.286 & 0.420 & $<.001$ & .056 & .792 \\
CMW & 17.4 & 34.5 & $<.001$ & .005 & .49 \\
BVR & 2.44 & 0.92 & $<.001$ & .002 & .106 \\
THW & 7.6 & 12.5 & $<.001$ & .002 & .026 \\
CTR & 2.33 & 2.86 & $<.001$ & $<.001$ & .235 \\
EI & 0.293 & 0.384 & $<.001$ & $<.001$ & .971 \\
FHVD & 25.7 & 38.8 & $<.001$ & $<.001$ & .059 \\
CA & 116.6 & 74.7 & $<.001$ & $<.001$ & .001 \\
SVW & 39.6 & 30.9 & $<.001$ & .288 \\
\hline
\end{tabular}

Note:-HC indicates healthy control; iNPH, idiopathic normal pressure hydrocephalus.

a Please refer to the Results section for detailed explanation. Two-way repeated measures ANOVA: angulation is a within-subjects factor (Angulation), and disease status is a between-subjects factor (iNPH/HC). Angulation by disease status interaction term (Angulation iNPH/HC) is significant for THW and CA only, with larger effects observed in the iNPH group for both biomarkers. Data are sorted in descending angulation $P$ value order (column 5: "Angulation"). Decreasing $P$ values indicate increasing statistical differences between measurements obtained at different section planes.

Table 2: Pair-wise comparisons between different planes performed with Bonferroni correction for biomarkers showing significant dependence on angulation $(P<.05)$ in the repeated measures ANOVA (as per Table 1) ${ }^{a}$

\begin{tabular}{|c|c|c|c|c|c|c|}
\hline Biomarker & $\begin{array}{c}\text { Bi-Call, } \\
\text { Bi-Comm }\end{array}$ & $\begin{array}{l}\text { Bi-Call, } \\
\mathrm{Hy}-\mathrm{Fa}\end{array}$ & $\begin{array}{l}\text { Bi-Call, } \\
\text { PBSM }\end{array}$ & $\begin{array}{l}\text { Bi-Comm, } \\
\text { Hy-Fa }\end{array}$ & $\begin{array}{l}\text { Bi-Comm, } \\
\text { PBSM }\end{array}$ & $\begin{array}{l}\text { Hy-Fa, } \\
\text { PBSM }\end{array}$ \\
\hline DESH & NS & NS & NS & NS & NS & NS \\
\hline $\operatorname{simpCA}$ & NS & NS & NS & NS & NS & NS \\
\hline FHD & NS & NS & NS & NS & NS & NS \\
\hline ZEl & NS & NS & NS & NS & NS & NS \\
\hline CMW & NS & NS & NS & NS & NS & NS \\
\hline BVR & NS & $\begin{array}{c}.007 \\
-0.04 \pm 0.01\end{array}$ & $\begin{array}{c}.009 \\
0.07 \pm 0.02\end{array}$ & NS & NS & $\begin{array}{c}<.001 \\
0.11 \pm 0.02\end{array}$ \\
\hline THW & NS & NS & NS & $\begin{array}{c}.002 \\
0.24 \pm 0.06\end{array}$ & $\begin{array}{c}.014 \\
0.32 \pm 0.10\end{array}$ & NS \\
\hline CTR & NS & NS & NS & NS & $\begin{array}{c}.004 \\
-0.12 \pm 0.03\end{array}$ & NS \\
\hline El & NS & NS & $\begin{array}{c}<.001 \\
-0.005 \pm 0.001\end{array}$ & NS & $\begin{array}{c}<.001 \\
-0.005 \pm 0.001\end{array}$ & $\begin{array}{c}<.001 \\
-0.005 \pm 0.001\end{array}$ \\
\hline FHVD & NS & NS & $\begin{array}{c}.014 \\
-0.52 \pm 0.17\end{array}$ & NS & $\begin{array}{c}<.001 \\
-0.92 \pm 0.20\end{array}$ & $\begin{array}{c}<.001 \\
-0.79 \pm 0.17\end{array}$ \\
\hline CA & $\begin{array}{c}.013 \\
-1.94 \pm 0.61\end{array}$ & NS & $\begin{array}{c}<.001 \\
-7.06 \pm 0.87\end{array}$ & NS & $\begin{array}{c}<.001 \\
-9.01 \pm 1.07\end{array}$ & $\begin{array}{c}<.001 \\
-8.24 \pm 0.91\end{array}$ \\
\hline SVW & $\begin{array}{c}.034 \\
-0.45 \pm 0.16\end{array}$ & NS & $\begin{array}{c}.005 \\
0.97 \pm 0.28\end{array}$ & NS & $\begin{array}{c}<.001 \\
1.42 \pm 0.30\end{array}$ & $\begin{array}{c}.001 \\
1.26 \pm 0.32\end{array}$ \\
\hline
\end{tabular}

Note:-Bi-Call indicates bicallosal; Bi-Comm, bicommissural; Hy-Fa, hypophysis-fastigium; PBSM, posterior brain stem margin; NS, not significant.

a $\mathrm{P}$ values (upper digit) and means \pm standard errors of paired differences are calculated $(n=80)$ and presented for those paired tests that are statistically significant.

\section{DISCUSSION}

Various linear, angular, volumetric, or index measurements have been proposed as radiologic biomarkers of iNPH in multiple studies. ${ }^{3,5-10,12,13,15,16}$ However, consensus on which biomarker is the most sensitive, specific, and accurate has not been reached.

Much less attention has been paid to standardization of biomarker measurements and their reliability with respect to imaging plane angulation. Ishii et $\mathrm{al}^{5}$ arbitrarily decided to use the bicommissural plane for CA measurements, stating that CAs varied on each plane, but they did not provide any further details. Cagnin et $\mathrm{al}^{6}$ tested callosal angle variability with respect to different section angulations on a limited group of 5 subjects. Toma et $\mathrm{al}^{9}$ showed that the EI varied depending on both the CT section level and angulation in a study of 10 patients with NPH; the researchers concluded that comparing the EI on CT scans obtained at different institutions using different protocols may result in inaccurate assessment of disease progression. Nonetheless, systematic comparison of biomarker variability with respect to different imaging plane angulations or robustness against angulation-related bias has not been published.

\section{Angulation-Related Variability}

Our results show that both the EI and CA, the most frequently used biomarkers for iNPH assessment, are subject to significant systematic differences when assessed on sections of different angulations. Because most CT and MR imaging brain studies primarily do not contain coronal sections aligned perpendicular to the bicommissural line, precise CA values cannot be obtained unless a $3 \mathrm{D}$ set of volumetric data is available and supplementary multiplanar reformatting is performed. This requirement is easily 
achievable in properly and prospectively designed research studies such as Miskin et al, ${ }^{3}$ Yamada et al, ${ }^{7}$ and Agerskov et al; ${ }^{22}$ however, it does not comply with the reality of routine clinical practice in which the CA is frequently measured on the readily available, yet inappropriately angulated, coronal section (typically the PBSM). According to our results, this issue may lead to incorrect diagnostic conclusions. As shown in Fig 3, the CA value correctly obtained from the coronal section perpendicular to the bicommissural line is pathologically decreased to $69^{\circ}$ in this subject, whereas the value obtained from the readily available "standard" coronal section aligned parallel to the PBSM yields $103^{\circ}$, which may be misinterpreted as a normal finding.

Similarly, if EI values are compared on cross-sectional studies of the same subject using various definitions of transverse planes, the differences obtained may be subject to a methodologic error rather than to the natural evolution of ventriculomegaly. This phenomenon might have contributed to the failure of the EI to correctly identify subjects with iNPH in a large study of 390 patients in which CT and MR imaging studies from 5 institutions using different protocols were retrospectively evaluated. ${ }^{8}$ Although the EI may show significant differences when measured on variously angulated transverse sections, the exact definition of the transverse plane used for EI evaluation has been missing in multiple research studies published such as Kojoukhova et $\mathrm{al}^{8}{ }^{8} \mathrm{Bao}$ et $\mathrm{al},{ }^{10}$ and Reinard et al. ${ }^{11}$ It remains debatable whether small statistical differences found in the EI are relevant in the setting of daily clinical routine; nonetheless, they may be of significance at least in those cases in which the EI approaches the cutoff value.

On the contrary, DESH, simplified CA, FHD, ZEI, and CMW did not show statistically significant differences related to varying section angulation (Tables 1 and 2) and proved to be insensitive to plane-tilting differences, which would predispose them better for comparing iNPH imaging studies performed at different institutions using different protocols. DESH proved to be the biomarker least prone to angulation-related variability, which might be attributed to its semiquantitative nature based on visual relative assessment of subarachnoid spaces.

The FHD also showed insignificant variability of results obtained at various section angulations. Owing to the lateral convex geometry of the frontal horns, the maximum FHD would remain unchanged over a wide range of section angulations. Besides, the FHD scored comparably with the EI in a recent study on the diagnostic performance of $15 \mathrm{iNPH}$ biomarkers. ${ }^{21}$ This finding has further raised the question of whether a simple linear measure such as the FHD could possibly replace the more complicated index measurement of the EI which, in addition, shows significant variability when measured on different transverse sections. The reason for the increased EI variability is that although its numerator (FHD) remains constant at the level of maximum frontal horn diameter, the denominator (inner skull diameter) varies on differently angulated sections, which all intersect at the FHD level. As shown in the example in Fig 3, the FHD remains the same at all angulations $(48.5 \mathrm{~mm})$, whereas the inner skull diameter ranges between 127.6 and $134.8 \mathrm{~mm}$.

Unlike the EI, the ZEI has not shown significant variability when assessed on different section planes using repeated measures ANOVA. Furthermore, the ZEI has shown better discriminatory power between subjects with iNPH and HCs than the EI in the recent study. ${ }^{21}$

When comparing the $\mathrm{CA}^{5}$ with the simplified $\mathrm{CA}^{6}$, the former (assessed at the posterior commissure) shows substantially increased variability at different angulations. This is because the angle formed by the medial walls of the lateral ventricles progressively varies near the ventricular trigones (and posterior commissure) but is relatively constant at the midportion of the corpus callosum, making the simplified CA at this location less susceptible to angulation-related deviations. Because the cerebral "uplift" and "wrapping" of the lateral ventricles around the posterior falx belong to the imaging hallmarks of iNPH, ${ }^{12,20,23}$ the changes of the ventricular geometry as well as the resulting CA angulation-related variability tend to be accentuated in subjects with iNPH. This tendency is in accordance with the analysis of the disease status interaction term in which the dependence on angulation has differed between the iNPH and HC groups, most significantly for CA $(P<.001)$, with larger effects observed in the iNPH group (Table 1, column "Angulation iNPH/HC"). Post hoc analysis within the iNPH group showed that the SD of CA values obtained on 4 coronal planes yielded $10.3^{\circ}$ (intraindividual differences of CA values ranged between $2^{\circ}$ and $40^{\circ}$ ), whereas the $\mathrm{SD}$ within the $\mathrm{HC}$ group was significantly lower $\left(5.8^{\circ}\right.$; intraindividual range, $\left.1^{\circ}-26^{\circ}\right)$.

Coronal sections aligned parallel with the brain stem vertical line (PBSM) showed the highest CA deviations from the prescribed bicommissural plane. Considering that the PBSM serves as the standard coronal imaging section at many institutions, improper-yet-not-rare assessment of the CA on PBSM sections may induce significant bias (Table 2 and Fig 3 ) and should be avoided. This observation should certainly not discourage radiologists from using the CA in routine practice because the CA has shown high discriminatory power between $\mathrm{HCs}$ and $\mathrm{iNPH}$, surpassing, for example, the EI or the simplified $\mathrm{CA},{ }^{21}$ yet it emphasizes that complying with the proper measurement methodology is essential.

\section{Study Limitations}

This study was not intended to suggest new imaging standards, evaluate the importance of radiologic biomarkers as supplementary tests for predicting outcome of CSF shunt surgery, or determine which plane or biomarker is the most accurate one. Instead, we have tried to investigate how different standards of section positioning affect individual iNPH biomarkers and whether inappropriate cross-sectional plane angulation may significantly influence iNPH biomarker measurements.

The diagnosis of iNPH was established on the basis of the international iNPH guideline; ${ }^{18}$ therefore, the EI of $>0.3$ was already used as a preselecting criterion. On the contrary, ventriculomegaly and EI increase are common in the overall elderly population, ${ }^{4}$ which enhances the diagnostic benefits of more iNPH-specific biomarkers such as the ZEI, DESH, or CA.

The orbitomeatal plane was not included in the assessment, possibly being viewed as a limitation of the study, but there were several reasons for this exclusion: Its use has gradually decreased with advancements of multidetector CT scanner technology, offering unprecedented multiplanar reformatting capabilities yet restricting gantry tilt options. Also, this plane cannot be precisely defined on crosssectional MR images, reducing its reproducibility on MR imaging. 
Our study cohort represented a highly selective sample of the elderly population comprising subjects with iNPH or HCs only. However, systematic comparison of angulation-related biomarker variability, which was the main objective of this study, should not be significantly influenced by this study design.

\section{CONCLUSIONS}

Seven of 12 iNPH biomarkers studied, including the frequently used EI and CA, show statistically significant deviations when measured on sections whose angulation differs from the original section definition. Strict adherence to the originally prescribed methodology of iNPH biomarker assessment (such as measuring the CA strictly on the coronal section oriented perpendicular to the bicommissural line) is considered essential to avoid incorrect diagnostic results. Volume acquisition of a 3D image dataset should be considered the standard of care in assessing patients for iNPH (as well as other dementias) on both CT and MR imaging because it provides the opportunity for reformatting correctly angulated section planes, thus complying with the proper measurement technique for each particular biomarker.

More radiologic as well as research attention should be paid to those biomarkers, which are the least sensitive to angulationrelated differences such as DESH, ZEI, or CMW; in addition, these biomarkers have also shown high discriminatory power between iNPH and HCs in a recently published study. ${ }^{21}$ If further research proves that these biomarkers show high diagnostic accuracy for iNPH as well as insensitivity to angulation-related bias, it would yield benefits for daily radiologic practice and potentially aid in designing the future versions of iNPH diagnostic guidelines.

Different institutions worldwide use various definitions of transverse and coronal brain imaging sections. Comparing CT or MR imaging studies from different sources using different protocols is not only arduous and less precise but may also lead to biased results when sections with improper angulation are used for assessment of specific biomarkers. Viewed from a broader perspective, a systematic effort to standardize and unify cross-sectional imaging plane definitions would bring substantial benefits extending far beyond the radiologic community.

Disclosures: Jonatan Salzer-UNRELATED: Consultancy: Mabion SA, Comments: consultancy fees*; Grants/Grants Pending: Synapsys, Interacoustics, Comments: material research support.* *Money paid to the institution.

\section{REFERENCES}

1. Brean A, Eide PK. Prevalence of probable idiopathic normal pressure hydrocephalus in a Norwegian population. Acta Neurol Scand 2008;118:48-53 CrossRef Medline

2. Isaacs AM, Riva-Cambrin J, Yavin D, et al. Age-specific global epidemiology of hydrocephalus: systematic review, metanalysis and global birth surveillance. PLoS ONE 2018;13:e0204926 CrossRef Medline

3. Miskin N, Patel H, Franceschi AM, et al. Alzheimer's Disease Neuroimaging Initiative. Diagnosis of normal-pressure hydrocephalus: use of traditional measures in the era of volumetric MR imaging. Radiology 2017;285:197-205 CrossRef Medline

4. Ambarki $K$, Israelsson $H$, Wåhlin $A$, et al. Brain ventricular size in healthy elderly: comparison between Evans Index and volume measurement. Neurosurgery 2010;67:94-99 CrossRef Medline

5. Ishii K, Kanda T, Harada A, et al. Clinical impact of the callosal angle in the diagnosis of idiopathic normal pressure hydrocephalus. Eur Radiol 2008;18:2678-83 CrossRef Medline
6. Cagnin A, Simioni M, Tagliapietra M, et al. A simplified callosal angle measure best differentiates idiopathic-normal pressure hydrocephalus from neurodegenerative dementia. J Alzheimers Dis 2015;46:1033-38 CrossRef Medline

7. Yamada S, Ishikawa M, Yamamoto K. Optimal diagnostic indices for idiopathic normal pressure hydrocephalus based on the 3D quantitative volumetric analysis for the cerebral ventricle and subarachnoid space. AJNR Am J Neuroradiol 2015;36:2262-69 CrossRef Medline

8. Kojoukhova M, Koivisto AM, Korhonen R, et al. Feasibility of radiological markers in idiopathic normal pressure hydrocephalus. Acta Neurochir 2015;157:1709-18 CrossRef Medline

9. Toma AK, Holl E, Kitchen ND, et al. Evans' index revisited: the need for an alternative in normal pressure hydrocephalus. Neurosurgery 2011;68:939-44 CrossRef Medline

10. Bao J, Gao Y, Cao Y, et al. Feasibility of simple linear measurements to determine ventricular enlargement in patients with idiopathic normal pressure hydrocephalus. J Craniofac Surg 2016;27:e462-65 CrossRef Medline

11. Reinard K, Basheer A, Phillips S, et al. Simple and reproducible linear measurements to determine ventricular enlargement in adults. Surg Neurol Int 2015;6:59 CrossRef Medline

12. Yamada S, Ishikawa M, Yamamoto K. Comparison of CSF distribution between idiopathic normal pressure hydrocephalus and Alzheimer disease. AJNR Am J Neuroradiol 2016;37:1249-55 CrossRef Medline

13. Sasaki M, Honda S, Yuasa T, et al. Narrow CSF space at high convexity and high midline areas in idiopathic normal pressure hydrocephalus detected by axial and coronal MRI. Neuroradiology 2008;50:11722 CrossRef Medline

14. Bradley WG Jr. CSF flow in the brain in the context of normal pressure hydrocephalus. AJNR Am J Neuroradiol 2015;36:831-38 CrossRef Medline

15. Benedetto N, Gambacciani C, Aquila F, et al. A new quantitative method to assess disproportionately enlarged subarachnoid space (DESH) in patients with possible idiopathic normal pressure hydrocephalus: the SILVER index. Clin Neurol Neurosurg 2017;158:27-32 CrossRef Medline

16. Evans WA Jr. An encephalographic ratio for estimating ventricular enlargement and cerebral atrophy. Arch NeurPsych 1942;47:931-37

17. Synek V, Reuben JR, Du Boulay GH. Comparing Evans' index and computerized axial tomography in assessing relationship of ventricular size to brain size. Neurology 1976;26:231-33 CrossRef Medline

18. Relkin N, Marmarou A, Klinge P, et al. Diagnosing idiopathic normal-pressure hydrocephalus. Neurosurgery 2005;57:S4-16 CrossRef Medline

19. Kitagaki H, Mori E, Ishii K, et al. CSF spaces in idiopathic normal pressure hydrocephalus: morphology and volumetry. AJNR Am J Neuroradiol 1998;19:1277-84 Medline

20. Hashimoto M, Ishikawa M, Mori E, et al. Study of INPH on neurological improvement (SINPHONI). Diagnosis of idiopathic normal pressure hydrocephalus is supported by MRI-based scheme: a prospective cohort study. Cerebrospinal Fluid Res 2010;7:18 CrossRef Medline

21. Ryska P, Slezak O, Eklund A, et al. Radiological markers of idiopathic normal pressure hydrocephalus: relative comparison of their diagnostic performance. J Neurol Sci 2020;408:116581 CrossRef Medline

22. Agerskov S, Wallin M, Hellström P, et al. Absence of disproportionately enlarged subarachnoid space hydrocephalus, a sharp callosal angle, or other morphologic MRI markers should not be used to exclude patients with idiopathic normal pressure hydrocephalus from shunt surgery. AJNR Am J Neuroradiol 2019;40:74-79 CrossRef Medline

23. Virhammar J, Laurell K, Cesarini KG, et al. The callosal angle measured on MRI as a predictor of outcome in idiopathic normal-pressure hydrocephalus. J Neurosurg 2014;120:178-84 CrossRef Medline 